\title{
Tuberculose: conhecimento e adesão às medidas profiláticas em indivíduos contatos da cidade do Recife, Pernambuco, Brasil
}

\section{Tuberculosis: knowledge and adherence to prophylactic measures in contact individuals of the city of Recife, Pernambuco, Brazil}

\author{
Amanda Queiroz Teixeira' (D), Isabella Chagas Samico² (D), Ariane Bezerra Martins (D), \\ Jadson Mendonça Galindo ${ }^{3}$ (D), Rosana de Albuquerque Montenegro ${ }^{4}$ (D), \\ Haiana Charifker Schindler ${ }^{4}$ (I) \\ 1 Prefeitura de Recife - Recife (PE), Brasil. \\ ${ }^{2}$ Grupo de Estudos de Gestão e Avaliação em Saúde, Instituto de Medicina Integral Prof. Fernando Figueira - IMIP - Recife (PE), \\ Brasil. \\ ${ }^{3}$ Hospital das Clínicas e Estado - Recife (PE), Brasil. \\ ${ }^{4}$ Instituto Aggeu Magalhães (FIOCRUZ) - Recife (PE), Brasil.
}

Como citar: Teixeira AQ, Samico IC, Martins AB, Galindo JM, Montenegro RA, Schindler HC. Tuberculose: conhecimento e adesão às medidas profiláticas em indivíduos contatos da cidade do Recife, Pernambuco, Brasil. Cad Saúde Colet, 2020;28(1):116-129. https://doi.org/10.1590/1414-462X202028010332

\section{Resumo}

Introdução: Para o controle da tuberculose, é fundamental interromper a cadeia de transmissão da doença. O Ministério da Saúde preconiza que 100\% dos contatos sejam examinados e iniciem tratamento da Infecção Latente por Mycobacterium tuberculosis. Nesse sentido, o conhecimento sobre a doença e a adesão à profilaxia por parte desses contatos são fatores que podem interferir no adequado controle da tuberculose. Objetivo: Descrever o conhecimento dos contatos de portadores de tuberculose sobre a doença e sua adesão às medidas profiláticas no Distrito Sanitário II em Recife/PE. Método: Estudo quantitativo, descritivo, utilizando questionários padronizados, aplicados a 140 contatos de tuberculose notificados de janeiro a dezembro de 2015. Análise dos dados realizada por meio de frequências simples. Resultados: Dentre os entrevistados, 75,7\% eram do sexo feminino, 55\% pardos, com baixos níveis de escolaridade e renda familiar. Destes, $84,3 \%$ acreditam que a tuberculose é grave, $48,6 \%$ consideram que a transmissão se faz compartilhando utensílios. Apenas $55 \%$ foram convidados para serem examinados e $76 \%$ referiram não saber que deveriam ir à consulta ou a importância desta. Conclusão: Os contatos de tuberculose possuem precário conhecimento sobre a doença, baixa adesão à atenção primária à saúde e a busca ativa dos contatos ainda é ineficiente.

Palavras-chave: tuberculose; prevenção de doença; adesão; conhecimentos, atitudes e prática em saúde; atenção primária à saúde.

\begin{abstract}
Background: For the control of tuberculosis, it is essential to interrupt its chain of transmission. The Ministry of Health recommends $100 \%$ of contacts being examined and initiated treatment of the Latent Mycobacterium tuberculosis infection. In this sense, the knowledge about the disease and adherence to prophylaxis by these contacts are factors that can interfere in the adequate control of tuberculosis. Objective: To describe the knowledge of the contacts of tuberculosis patients on the disease and their adherence to prophylactic measures in the Sanitary District II in Recife / PE. Method: A quantitative and descriptive study was carried out using standardized questionnaires, applied to
\end{abstract}

Trabalho realizado no Distrito Sanitário II - Recife (PE), Brasil.

Correspondência: Haiana Charifker Schindler. E-mail: haia@cpqam.fiocruz.br

Fonte de financiamento: nenhuma

Conflito de interesses: nada a declarar.

Recebido em: Ago. 10, 2018. Aprovado em: Jun. 01, 2019.
Este é um artigo publicado em acesso aberto (Open Access) sob a licença Creative Commons Attribution, que permite uso, distribuição e reprodução em qualquer meio, sem restrições desde que o trabalho original seja corretamente citado. 
140 contacts of tuberculosis notified from January to December 2015. Data analysis carried out through simple frequencies. Results: Among the interviewees, $75.7 \%$ were female, 55\% brown, with low levels of schooling and family income; of these $84.3 \%$ believe that tuberculosis is serious, $48.6 \%$ consider that transmission is done by sharing utensils. Only $55 \%$ were invited to be examined and $76 \%$ reported not knowing they should go to the consultation or the importance of these. Conclusion: The contacts of tuberculosis have poor knowledge about the disease, low adherence to primary health care and the active search for contacts is still inefficient.

Keywords: tuberculosis; prevention of diseases; knowledge, attitudes and practice in health; primary health care.

\section{INTRODUÇÃO}

A tuberculose (TB) é uma doença infecciosa, causada por sete espécies do Complexo Mycobacterium tuberculosis (CMtb), que podem afetar o homem e outros mamíferos. Mundialmente considerada um importante problema de saúde pública, segundo a Organização Mundial de Saúde (OMS), estima-se que 1,7 bilhão de pessoas estejam infectadas. Contudo, as pessoas vivendo com HIV, ou fatores de risco a exemplo do tabagismo, da desnutrição, do alcoolismo e da diabetes, possuem maior probabilidade de desenvolver a doença em algum momento da vida ${ }^{1,2}$.

Na Assembleia Mundial de Saúde das Nações Unidas, junto à OMS em 2014, foi aprovada a nova estratégia mundial para enfrentamento da $T B$, visando a um mundo livre da doença até 2050. Recentes análises demonstram que uma das melhores estratégias para o alcance dessa meta é a identificação e o tratamento dos contatos portadores da Infecção Latente por Mycobacterium tuberculosis (ILTB) 3 .

A OMS, em 2017, estimou 282 mil novos casos de TB nas Américas. No Brasil, a TB possui profundas raízes sociais. As desigualdades e as condições de saúde das populações continuam como agravantes. As implicações epidemiológicas e sociais apresentadas pela TB necessitam de medidas de prevenção e controle $e^{3,5}$.

No grupo dos 30 países priorizados pela OMS, por concentrar $80 \%$ do total de casos de tuberculose no mundo, o Brasil ocupa a vigésima posição em números absolutos de casos. Contudo, tem apresentado decréscimo na incidência da tuberculose e no número de casos novos por 100 mil habitantes. Nos últimos anos, houve uma redução de 9,05\%, passando de 38,8 casos/100 mil habitantes em 2006 para 35,2 casos/100 mil habitantes em 20173.

Apesar dessa melhora, ainda restam desafios para a redução do número de casos da doença. No estado de Pernambuco e sua capital, Recife, o coeficiente de incidência foi de 46 e 85,5/100.000 habitantes, respectivamente, em 20173,6.

Em 2017, o Ministério da Saúde (MS), conforme a Estratégia pelo Fim da Tuberculose da Organização Mundial de Saúde (OMS), elaborou o Plano Nacional pelo Fim da Tuberculose como Problema de Saúde Pública. Apresenta como meta reduzir os coeficientes de mortalidade e de incidência, comparados aos dados de 2015, até $2035^{6}$.

Para o controle da tuberculose é fundamental interromper a cadeia de transmissão da doença. Cada pessoa com TB pulmonar não diagnosticada tende a infectar de 10 a 15 pessoas/ano, e, destas, uma a duas adoecem, mantendo a transmissão e a doença em nível de endemia7. O indivíduo com tuberculose pulmonar ativa, ao tossir, espirrar ou falar, libera gotículas (gotículas de Pflüger) que transportam os bacilos para o ambiente. Quanto menores essas gotículas (núcleos de Wells), mais tempo elas permanecem no ar, e, portanto, maior a possibilidade de que sejam aspiradas, inaladas e infectem outras pessoas ${ }^{8}$.

$\mathrm{O}$ caso de tuberculose identificado inicialmente como sendo novo ou recorrente, em pessoa de qualquer idade, em uma casa específica, é considerado um caso índice. Além da importância do início do tratamento precoce, o caso índice deve ser investigado para identificação das pessoas que serão consideradas contatos².

"Household contact" é um termo em inglês cuja melhor tradução para o português é "contato domiciliar". É usado para definir os contatos de pacientes com TB que convivem com o caso índice na mesma residência. "Close contact" é o termo em inglês cuja tradução é"contato próximo". É usado em vários trabalhos para definir os contatos próximos do caso índice, mas 
não necessariamente os domiciliares. Inclui também parentes que não moram na mesma casa, colegas de trabalho, de atividades de lazer e outros tipos de contatos ${ }^{10}$.

As informações sobre os contatos e o tipo de convívio estabelecido devem ser listadas e, sempre que possível, deve-se realizar visita domiciliar para melhor entendimento das circunstâncias e convidá-los a comparecer à Unidade de Saúde para serem avaliados e, se necessário, solicitar exames, como radiografia de tórax, baciloscopia e prova tuberculínica (PT) com PPD (Derivado Proteico Purificado) ou do Interferon-Gamma Release Assays (IGRA) ${ }^{11}$. A realização da PT ou IGRA, nos contatos assintomáticos de TB, favorece o diagnóstico da ILTB, que é definida como sendo a presença de $M$. tuberculosis no organismo com ausência de manifestações clínicas ${ }^{2,11}$.

O Ministério da Saúde (MS) preconiza que 100\% dos contatos identificados sejam examinados e que se inicie o tratamento da ILTB com a finalidade de reduzir o risco de adoecimento. Atualmente, no Brasil, recomenda-se o tratamento com a Isoniazida $(\mathrm{H})$ ou a utilização da Rifampicina $(\mathrm{R})^{3,11}$.

A oportunidade de prevenir a progressão da doença ativa entre os contatos com ILTB tem sido comprovada em diversas pesquisas. O tratamento da TB latente gera impacto positivo nas taxas de incidência. Contudo, ainda existem falhas na investigação e condução dos casos pelos profissionais ${ }^{4}$. Estudos realizados em distritos do sul da Índia concluíram que, sob condições programadas, não existe contato que tenha sido submetido à ótima execução da triagem e aplicação de rotina profilática de Isoniazida $(\mathrm{H} / 100 \mathrm{mg})$ devido ao não seguimento das recomendações do Programa de Controle da Tuberculose Nacional por parte dos profissionais, quanto ao rastreio e tratamento dos $\operatorname{casos}^{12}$. Demonstraram, ainda, que o diagnóstico dos contatos domiciliares de tuberculose, aos quais se realizam visitas domiciliares, é substancialmente maior do que a detecção passiva de $\operatorname{casos}^{13}$.

Em Salvador, Bahia, 269 contatos domiciliares de casos índices de tuberculose foram acompanhados por 12 meses. A prevalência da doença na população estudada foi de 3,7\% e a prevalência de infecção, após o período de 12 meses de seguimento, de 63,9\%. Concluiu-se que os contatos domiciliares de pacientes com tuberculose pulmonar ativa têm alto risco de infecção ${ }^{14}$.

É importante destacar que a adesão aos programas de prevenção ainda é baixa em todo o mundo, e que é fundamental excluir a doença em atividade antes da indicação do tratamento da ILTB ${ }^{15}$.

A adesão é um termo utilizado em diferentes contextos. A liberdade do indivíduo em escolher ou não determinada orientação, assumindo responsabilidades sobre seu papel, dentro das suas crenças e valores em relação ao processo saúde-doença, é relevante ${ }^{16}$. Nesse momento, tem-se de analisar como o paciente se percebe. A exemplo da AIDS, na qual a ausência de sintomas em algumas fases do processo de adoecimento é um dos fatores citados para a não adesão ao tratamento ${ }^{17}$.

A interpretação do processo saúde-doença é conduzida de acordo com o entendimento individual. A vivência do indivíduo junto à sociedade possibilita que o mesmo tenha atitudes de conformismo ou enfrentamento, bem como aderir a algo ou não. Diante das suas experiências, em uma perspectiva ativa frente às adversidades, formam-se o compromisso e o sujeito do processo, não mais o indivíduo cumpridor das orientações terapêuticas que não correspondem às suas necessidades essenciais ${ }^{18}$.

O conhecimento da população acerca da tuberculose é um fator relevante na identificação da doença e para as ações a serem desenvolvidas. Em um estudo realizado em 2012, na cidade de Natal/RN, evidenciou-se um conhecimento satisfatório das famílias sobre a TB. Contudo, a compreensão da transmissibilidade e dos cuidados mostrou-se preocupante, por acreditarem na disseminação da doença por meio de compartilhamento de utensílios. Constatou-se ainda que os indivíduos de baixa renda apresentavam menores chances de conhecimento sobre a patologia ${ }^{19}$.

Dessa forma, faz-se necessário explicitar a vulnerabilidade em que os indivíduos contatos, de um caso índice bacilífero, possuem. As chances de exposição das pessoas ao adoecimento, resultante de aspectos não só individuais, mas também coletivos e contextuais, os tornam 
vulneráveis, mais suscetíveis às infecções e ao adoecimento ${ }^{11,20}$. Nessa camada social, a proximidade do convívio devido a estrutura precária da casa, ausência de ventilação adequada, umidade e famílias numerosas propicia o contágio ${ }^{21}$.

Os avanços na interrupção da cadeia de transmissão da tuberculose vão além das ações curativas. É fundamental uma abordagem mais ampla, analisando o contexto sociodemográfico até a incorporação de ações preventivas empregadas na Atenção Primária à Saúde (APS).

Este estudo objetivou descrever o conhecimento dos contatos de portadores de TB sobre a doença e a sua adesão às medidas profiláticas.

\section{MÉTODO}

Trata-se de estudo descritivo, a partir de dados secundários dos casos índices de tuberculose coletados no Sistema de Informação de Agravos e Notificação (Sinan) e dados primários obtidos por meio de entrevista com os indivíduos contatos de tuberculose no Distrito Sanitário II (DS II) da cidade do Recife.

Recife, capital do estado de Pernambuco, em 2015, possuía uma população estimada em aproximadamente 1.608.488 habitantes. Administrativamente, possui divisão geográfica e sanitária, de oito Distritos Sanitários (DS) 22,23.

Desses, os DS II, IV e V são os que apresentam maior incidência de tuberculose, segundo dados epidemiológicos da Secretaria Municipal de Saúde ${ }^{5}$. Contudo, os DS IV e V possuem unidades prisionais em seu território e essas elevam o número de casos. Assim, esse estudo foi realizado no DS II por possuir maior número de registros de casos de TB em uma população não pertencente ao sistema prisional.

Entre 02 de janeiro e 31 de dezembro de 2015, foram registrados, no DS II, exclusivamente pelas Equipes de Saúde da Família (ESF), 486 indivíduos contatos de TB pulmonar, e desses, apenas $240(39,6 \%)$ foram examinados ${ }^{23}$. O estudo foi realizado com os contatos intradomiciliares dos casos índices de tuberculose notificados pelas ESF do DS II, no Sinan. Esse período foi definido objetivando evitar o viés de memória.

Depois de identificados os casos índices e seus contatos intradomiciliares, houve um sorteio dos casos índices, com amostragem por conglomerado, por probabilidade proporcional, em que o caso índice se caracterizou como sua unidade primária de amostragem e, esse mesmo caso índice, representou um conjunto de indivíduos contatos. Apenas os contatos a partir dos 10 anos de idade foram elegíveis a participar, devido à dificuldade em coletar informações acerca de conhecimento sobre tuberculose, em indivíduos com idade inferior a esta.

O número de casos sorteados foi estimado pela média de contatos por caso. Dos 146 casos de tuberculose, notificados exclusivamente pelas ESF, havia 486 contatos intradomiciliares registrados. Dividiu-se o número de contatos (486) pelo número de casos (146), obtendo-se a média de aproximadamente três contatos por caso a serem entrevistados.

Para estimar o tamanho da amostra, foram considerados os parâmetros: população de contatos de tuberculose notificados pelas ESF do DS II (Sinan), em 2015, de 486 indivíduos, prevalência esperada de $50 \%$ (o que maximiza a amostra), um erro aceitável de 7\%, com nível de confiança de $95 \%$, resultando em uma amostra de 140 contatos.

Para uma amostra de 140 contatos, foram sorteados 46 casos índices (140 divididos pela média de contatos de três, resultaram em 46 casos índices). A seleção foi aleatória com probabilidade proporcional ao número de contatos por caso. Os sorteios realizados utilizaram intervalo amostral para haver a equiprobabilidade.

As variáveis consideradas para o estudo foram: a) Sociodemográficas: sexo, faixa etária, raça/cor, religião, comorbidades (diabetes, HIV/AIDS, câncer, transplantado renal e uso de corticoide), fatores de risco (etilismo, tabagismo e drogas ilícitas), escolaridade (anos de estudo), renda familiar mensal, vínculo empregatício e/ou benefício social, número de pessoas residentes no domicílio, número de cômodos na casa, estrutura do domicílio (alvenaria, madeira, taipa ou papelão); b) Conhecimento sobre tuberculose: conceito/definição, forma de contágio, diagnóstico, tratamento, medidas profiláticas; c) Ações do Programa Nacional de Controle da Tuberculose (PNCT) e de adesão às medidas profiláticas: recebimento de visita do ACS e outro profissional de saúde acerca da tuberculose do caso índice, recebimento de orientações acerca 
da tuberculose por algum profissional de saúde da ESF, realização de exame físico pelo médico ou enfermeiro, solicitação de exames complementares, indicação para realizar tratamento da ILTB e o motivo do não comparecimento à consulta.

A coleta de dados foi realizada no domicílio dos contatos, no período de maio a agosto de 2016, com duração média de 10 minutos por questionário aplicado. Os questionários foram uma adaptação de instrumento utilizado em outro estudo realizado em $2010^{24}$.

As visitas foram agendadas previamente e realizadas pela pesquisadora principal acompanhada por um Agente Comunitário de Saúde (ACS) da área. Os indivíduos maiores de 10 anos e menores de 18 anos, responderam ao questionário mediante a presença de um responsável. Após a realização de cada entrevista, com todos os contatos do domicílio, foram fornecidas informações sobre tuberculose e contatos de TB. Dessa forma, em cada domicílio visitado, a informação sobre tuberculose foi oportunizada pela pesquisadora.

Para o processamento e análise dos dados, foi utilizada a plataforma do Tabwin 32, tabulando os casos no Sinan elegíveis e distribuição dos contatos. Usou-se o Epi Info 7.1.5.2 para cálculo da amostragem. Para o sorteio e as tabulações das variáveis, utilizou-se o programa EXCEL, versão 2010, e o software Statistical Package for the Social Sciences (IBM SPSS) versão 20, para categorização dos dados.

O projeto do estudo, consonante com a Resolução do Conselho Nacional de Saúde (CNS) $n^{0} 466,12$ de dezembro de 2012, foi aprovado pelo Comitê de Ética em Pesquisa do Centro de Pesquisas Aggeu Magalhães/Fiocruz sob o Parecer n ${ }^{0}$ 553416.1.0000.5190, em 28 de abril de 2016.

\section{RESULTADOS}

Na Tabela 1, apresentam-se os resultados relativos às características sociodemográficas dos entrevistados. A maioria (75,7\%) é do sexo feminino, parda (55\%), com 18 a 39 anos (37,9\%), possui entre quatro e sete anos de estudos (51,4\%), evangélica (37\%) e com renda familiar de 1 a 1,9 salário mínimo (47,8\%). Os domicílios possuem de duas a quatro pessoas por domicílio $(58,5 \%)$, residindo em casa com três a cinco cômodos $(45,7 \%)$, de alvenaria $(97,9 \%)$ (Tabela 2$)$.

Tabela 1. Distribuição das variáveis sociodemográficas dos contatos de tuberculose. DS II, Recife - PE, 2016

\begin{tabular}{|c|c|c|}
\hline Variáveis & $\mathbf{N}$ & $\%$ \\
\hline \multicolumn{3}{|l|}{ Sexo } \\
\hline Feminino & 106 & 75,7 \\
\hline Masculino & 34 & 24,3 \\
\hline \multicolumn{3}{|l|}{ Raça/Cor } \\
\hline Pardo & 77 & 55 \\
\hline Preto & 32 & 23 \\
\hline Branco & 21 & 15 \\
\hline Amarelo & 4 & 3 \\
\hline Indígena & 6 & 4 \\
\hline \multicolumn{3}{|l|}{ Faixa Etária } \\
\hline $10-17$ & 22 & 15,7 \\
\hline $18-39$ & 53 & 37,9 \\
\hline $40-59$ & 45 & 32,1 \\
\hline$>60$ & 20 & 14,3 \\
\hline \multicolumn{3}{|l|}{ Anos de estudos } \\
\hline Nenhum & 9 & 6,4 \\
\hline De 1 a 3 anos de estudos & 8 & 5,7 \\
\hline
\end{tabular}


Tabela 1. Continuação...

\begin{tabular}{|c|c|c|}
\hline Variáveis & $\mathbf{N}$ & $\%$ \\
\hline De 4 a 7 anos de estudos & 72 & 51,4 \\
\hline De 8 a 11 anos de estudos & 25 & 17,9 \\
\hline 12 ou mais anos de estudos & 26 & 18,6 \\
\hline \multicolumn{3}{|l|}{ Renda familiar } \\
\hline$<1$ salário & 42 & 30 \\
\hline De 1 - 1,9 salário & 67 & 47,8 \\
\hline De 2 - 2,9 salários & 19 & 13,6 \\
\hline >3 salários & 12 & 8,6 \\
\hline \multicolumn{3}{|l|}{ Religião } \\
\hline Sem religião & 49 & 35 \\
\hline Católica & 37 & 26 \\
\hline Evangélica & 51 & 37 \\
\hline Kardecista & 3 & 2 \\
\hline \multicolumn{3}{|c|}{ Número de pessoas por domicílio } \\
\hline $2-4$ pessoas & 82 & 58,5 \\
\hline 5 - 9 pessoas & 53 & 37,9 \\
\hline$>10$ pessoas & 5 & 3,6 \\
\hline \multicolumn{3}{|c|}{ Número de cômodos por domicílio } \\
\hline Entre 1 e 2 cômodos & 14 & 10 \\
\hline Entre 3 e 5 cômodos & 64 & 45,7 \\
\hline > 6 cômodos & 62 & 44,3 \\
\hline \multicolumn{3}{|l|}{ Estrutura do domicílio } \\
\hline Alvenaria & 137 & 97,9 \\
\hline Madeira & 3 & 2,1 \\
\hline TOTAL & 140 & 100 \\
\hline
\end{tabular}

Tabela 2. Distribuição das comorbidades e dos fatores comportamentais identificados nos contatos de TB. DS II, Recife - PE, 2016

\begin{tabular}{|c|c|c|}
\hline Variáveis & $\mathbf{N}$ & $\%$ \\
\hline \multicolumn{3}{|l|}{ Comorbidade* } \\
\hline Insuficiência Renal & 3 & 2,1 \\
\hline Câncer & 4 & 2,9 \\
\hline Uso de Corticoide & 9 & 6,4 \\
\hline Diabetes & 10 & 7 \\
\hline Nenhuma morbidade & 166 & 81,6 \\
\hline \multicolumn{3}{|l|}{ Fatores comportamentais* } \\
\hline Etilista de $>1$ copo um dia na semana & 45 & 32,1 \\
\hline Tabagista > 1 cigarro/dia & 27 & 19,3 \\
\hline Drogas ilícitas & 6 & 4,3 \\
\hline Nenhum fator comportamental & 78 & 44,3 \\
\hline TOTAL & 140 & 100 \\
\hline
\end{tabular}

*Múltiplas respostasa 
Em relação às comorbidades e aos fatores comportamentais, verifica-se que $7 \%$ dos contatos entrevistados são diabéticos e $32,1 \%$ são etilistas (Tabela 3).

Tabela 3. Conhecimento sobre tuberculose. DS II, Recife - PE, 2016

\begin{tabular}{|c|c|c|}
\hline Variáveis & $\mathbf{N}$ & $\%$ \\
\hline \multicolumn{3}{|l|}{ Tuberculose } \\
\hline Doença & 108 & 77,1 \\
\hline Não sei & 23 & 16,4 \\
\hline Outro & 9 & 6,5 \\
\hline \multicolumn{3}{|l|}{ É uma doença grave } \\
\hline Sim & 118 & 84,3 \\
\hline Não & 19 & 13,6 \\
\hline Não sei & 3 & 2,1 \\
\hline \multicolumn{3}{|l|}{ Sintomas* } \\
\hline Tosse & 102 & 73 \\
\hline Febre & 58 & 41 \\
\hline Perda de peso & 21 & 15 \\
\hline Sudorese noturna & 3 & 2 \\
\hline Falta de apetite & 14 & 10 \\
\hline Outros sintomas & 62 & 44 \\
\hline \multicolumn{3}{|l|}{ Transmissão } \\
\hline Sim & 132 & 94,3 \\
\hline Não & 8 & 5,7 \\
\hline \multicolumn{3}{|l|}{ Modo de transmissão* } \\
\hline Utensílios & 68 & 48,6 \\
\hline $\mathrm{Ar}$ & 32 & 22,9 \\
\hline Tosse & 18 & 12,9 \\
\hline Fala & 14 & 10 \\
\hline Espirro & 9 & 6,4 \\
\hline Saliva & 15 & 10,7 \\
\hline Não sei & 17 & 12,1 \\
\hline \multicolumn{3}{|l|}{ Cura } \\
\hline Sim & 135 & 96,4 \\
\hline Não & 4 & 2,9 \\
\hline Não sei & 1 & 0,7 \\
\hline \multicolumn{3}{|l|}{ Tratamento } \\
\hline Remédios diários & 113 & 80,7 \\
\hline Evitar frio & 1 & 0,7 \\
\hline Não sei & 26 & 18,6 \\
\hline \multicolumn{3}{|l|}{ Tempo de tratamento } \\
\hline 6 meses & 94 & 67 \\
\hline 01 ano & 6 & 4 \\
\hline Não sei & 40 & 29 \\
\hline TOTAL & 140 & 100 \\
\hline
\end{tabular}

*Múltiplas respostas 
Quanto ao conhecimento sobre tuberculose, $77,1 \%$ dos entrevistados referem que a TB é uma doença e $84,3 \%$ a consideram grave, e que apresenta como sintomas principais a tosse e outros sintomas ( $73 \%$ e $44 \%$, respectivamente). Acreditam que é transmissível $(94,3 \%$ ) e o maior meio de transmissão são os utensílios (48,6\%). É uma doença curável $(96,4 \%)$, com tratamento medicamentoso $(80,7 \%)$ por seis meses $(67 \%)$ (Tabela 4$)$.

Tabela 4. Conhecimento sobre profilaxia em tuberculose. DS II, Recife - PE, 2016

\begin{tabular}{|c|c|c|}
\hline Variáveis & $\mathbf{N}$ & $\%$ \\
\hline \multicolumn{3}{|l|}{ Tuberculose pode ser prevenida } \\
\hline Sim & 112 & 80 \\
\hline Não & 15 & 10,7 \\
\hline Não sei & 13 & 9,3 \\
\hline \multicolumn{3}{|l|}{ Como pode prevenir* } \\
\hline Separar os utensílios & 45 & 32,1 \\
\hline Melhoria da qualidade de vida & 19 & 13,6 \\
\hline Medicamentos & 9 & 6,4 \\
\hline Casa maior e arejada & 5 & 3,6 \\
\hline Ambiente limpo & 9 & 6,4 \\
\hline Não sei & 6 & 4,3 \\
\hline Outros & 19 & 13,6 \\
\hline \multicolumn{3}{|l|}{ Onde aprendeu sobre TB* } \\
\hline Amigos/Familiares & 76 & 54 \\
\hline Profissional de Saúde & 31 & 22 \\
\hline Escola & 12 & 9 \\
\hline Internet & 25 & 18 \\
\hline Outro & 3 & 2 \\
\hline TOTAL & 147 & 105 \\
\hline
\end{tabular}

*Múltiplas respostas

Sobre o conhecimento da profilaxia em tuberculose, verifica-se que $80 \%$ dos entrevistados acreditam que a TB pode ser prevenida. Desses, 32,1\% relatam que a prevenção se faz com a separação dos utensílios domésticos dos pacientes com tuberculose e $54 \%$ informaram que o seu conhecimento sobre tuberculose vem de amigos e familiares que já tiveram TB (Tabela 5).

Dos contatos entrevistados, $62,1 \%$ afirmaram que os profissionais de saúde não explicaram sobre tuberculose e $94 \%$ acreditam que deveriam ser convidados para realização de exames. Ainda, $55 \%$ desses entrevistados não compareceram à consulta justificando não saber que deveriam ir ou realizar exames. A adesão às consultas se deu em $61,4 \%$ dos indivíduos preocupados em pegar TB. E 75,4\% foram submetidos à radiografia de tórax para investigação de tuberculose. Retornaram com os resultados dos exames à unidade de saúde, $84,2 \%$ dos contatos examinados. Desses, foi indicado e iniciado, o tratamento da ILTB em $4,2 \%$. Dos entrevistados, $76 \%$ relataram que não compareceram à consulta por não saber que precisavam ir (Tabela 5). 
Tabela 5. Adesão às consultas para exames profiláticos em contados de TB. DS II - Recife - PE, 2016

\begin{tabular}{|c|c|c|}
\hline Variáveis & $\mathbf{N}$ & $\%$ \\
\hline \multicolumn{3}{|l|}{ Profissional explicou } \\
\hline Sim & 53 & 37,9 \\
\hline Não & 87 & 62,1 \\
\hline \multicolumn{3}{|l|}{ Contatos devem ser examinados } \\
\hline Sim & 131 & 94 \\
\hline Não & 9 & 6 \\
\hline \multicolumn{3}{|l|}{ Foi convidado para consulta } \\
\hline Sim & 77 & 55 \\
\hline Não & 63 & 45 \\
\hline \multicolumn{3}{|l|}{ Compareceu à consulta } \\
\hline Sim & 57 & 74 \\
\hline Não & 20 & 26 \\
\hline \multicolumn{3}{|l|}{ Motivação para ir à consulta* } \\
\hline Preocupado em pegar tuberculose & 35 & 61,4 \\
\hline Sentiu-se mal & 1 & 1,7 \\
\hline Profissional de saúde orientou & 31 & 54,38 \\
\hline Sabia da importância & 1 & 1,7 \\
\hline Não sei/Não tenho certeza & 2 & 3,5 \\
\hline \multicolumn{3}{|l|}{ Faltou trabalho } \\
\hline Sim & 8 & 14 \\
\hline Não & 49 & 86 \\
\hline
\end{tabular}

\section{RETORNO COM RESULTADOS}

\section{Exames solicitados*}

\begin{tabular}{lcc}
\hline Ausculta pulmonar & 13 & 22,8 \\
\hline Exame de sangue & 5 & 8,8 \\
\hline Radiografia de tórax & 43 & 75,4 \\
\hline Orientou sobre TB & 5 & 8,8 \\
\hline PPD & 7 & 12,2 \\
\hline Solicitação de BK & 27 & 47,4 \\
\hline Você voltou à unidade & & 84,2 \\
\hline Sim & 48 & 15,8 \\
\hline Não & 9 & \\
\hline Indicado tratamento & & 4,2 \\
\hline Sim & 2 & 95,8 \\
\hline Não & 46 & \\
\hline
\end{tabular}

*Múltipla escolha 
Tabela 5. Continuação...

\begin{tabular}{|c|c|c|}
\hline Variáveis & $\mathbf{N}$ & $\%$ \\
\hline \multicolumn{3}{|l|}{ Realizou tratamento } \\
\hline Sim & 2 & 4,2 \\
\hline Não se aplica & 46 & 95,8 \\
\hline \multicolumn{3}{|l|}{ Motivo do não comparecimento } \\
\hline Não sabia que deveria ir & 63 & 76 \\
\hline Não preciso ir/Não estou doente & 10 & 12 \\
\hline Muito ocupado & 4 & 4,8 \\
\hline Tempo de espera & 2 & 2,4 \\
\hline Localização da Unidade & 1 & 1,2 \\
\hline Ninguém para ir comigo & 3 & 3,6 \\
\hline TOTAL & 83 & 100 \\
\hline
\end{tabular}

*Múltipla escolha

\section{DISCUSSÃO}

Este estudo descreveu o conhecimento dos indivíduos contatos de tuberculose, sobre a doença e as possíveis causas para aderir ou não aos procedimentos profiláticos de triagem da infecção por TB. Os resultados encontrados sinalizam fragilidades acerca do conhecimento e da adesão às medidas profiláticas da doença.

Identificou-se que o perfil dos contatos são mulheres pardas, com menos de 40 anos, baixa escolaridade, residentes em casa de alvenaria, e que vivem em aglomerados humanos com renda mensal de um salário mínimo. Esses achados são semelhantes aos do estudo realizado no Rio Grande do Sul, que demonstrou a predominância de contatos do sexo feminino (50,6\%) na faixa etária entre 20 e 29 anos de idade ${ }^{8}$. Os baixos níveis de renda e escolaridade possibilitam o aumento da vulnerabilidade à tuberculose por refletir acesso individual e desigual à informação, advindo do conhecimento insuficiente ou inadequado sobre a doença ${ }^{24}$.

Quanto ao conhecimento sobre tuberculose, de forma geral, os entrevistados referem que sabem que a TB é grave, tem cura e tratamento. Entretanto, desconhecem a forma de transmissão e os demais sintomas. O saber sobre a doença é referido, por mais da metade dos participantes, por experiências junto a amigos ou familiares que já tiveram a doença. A informação sobre a tuberculose torna-se indispensável para o resgate do conhecimento acerca do processo saúde-doença de maneira a reduzir os estigmas sociais e desmistificar a doença ${ }^{19}$. Pode, inclusive, influenciar nas práticas e atitudes mais comuns dos contatos de pacientes com TB e na investigação dos entraves que os distanciam das consultas profiláticas ${ }^{25}$.

Também relacionada à adesão às medidas profiláticas, a situação socioeconômica interfere de forma significativa na realização do tratamento de TB, assim como a escolaridade, a concepção sobre o processo saúde-doença, os efeitos dos medicamentos tuberculostáticos, drogas lícitas e ilícitas ${ }^{26}$. Identificar e compreender as barreiras relacionadas à adesão podem direcionar as intervenções ${ }^{27}$.

No que concerne à profilaxia em tuberculose, verifica-se que os entrevistados acreditam que a TB pode ser prevenida separando os utensílios. Importante proporção afirmou que os contatos devem ser examinados, porém, pouco mais da metade dos entrevistados foram convidados. Destes, a maior motivação para ter comparecido às consultas foi o receio de infectar-se com TB. A radiografia de tórax foi o exame mais solicitado para avaliação desses, seguida pelo exame de escarro. Segundo o Ministério da Saúde (MS), para os maiores de 10 anos, o exame preconizado, prioritariamente, é o PPD (Derivado Proteico Purificado). 
Contudo, é importante destacar que, no ano de 2015, houve escassez temporária do PPD devido ao fechamento do laboratório que o produzia, na Dinamarca, e o Ministério da Saúde (MS) disponibilizou Nota Técnica para a condução e investigação dos contatos de tuberculose ${ }^{28}$. Achados semelhantes foram encontrados em estudo realizado no Vietnã, com 162 contatos de tuberculose, com idades entre 33 e 35 anos, registrados em 12 distritos locais, divididos de forma randomizada em casos e controles. Demonstraram que uma elevada proporção acreditava que a tuberculose poderia ser transmitida por meio do compartilhamento de utensílios, que os exames dos contatos são importantes e que a distância das clínicas/unidades é um fator dificultador ${ }^{25}$.

Nos EUA, em relátorio do CDC (Centers of Disease Control and Prevention), com os dados sobre investigações de contato dos 50 estados, no Distrito de Columbia (DC) e em Porto Rico, entre os anos de 2003 e 2012, constatou-se que, realizando a investigação dos contatos de TB, estratégia recomendada mundialmente, pôde-se verificar que aproximadamente $1 \%$ dos contatos apresentavam tuberculose. Ao aumentar a detecção de contatos com diagnóstico de ILTB e iniciar o tratamento, quando necessário, haveria redução considerável dos casos de TB. Estimou-se que 128 novos casos, em cinco anos, foram evitados, devido ao diagnóstico precoce e ao tratamento da ILTB, em 2012, e que, apesar das recomendações do tratamento medicamentoso para ILTB, mais de um terço desses contatos não foram submetidos aos exames e tratamento, possivelmente por falta de informação ${ }^{29}$. Em estudo recente realizado em três capitais brasileiras (Rio de Janeiro, Manaus e Recife), a respeito da transmissão e prevenção da TB, junto a profissionais médicos e enfermeiros da atenção básica, constatou-se que, mesmo havendo treinamento prévio, existem lacunas quanto ao conhecimento e à atitude acerca do tratamento dos contatos de tuberculose $e^{4}$.

Na Nigéria, um estudo revelou que muitas pessoas desconhecem sobre sinais e sintomas da tuberculose, e a infecção pelo bacilo. Sugere-se o esclarecimento sobre a doença como forma de melhor contribuição ${ }^{30,31}$. Em outro estudo, realizado na Etiópia, o nível de escolaridade e o conhecimento em tuberculose apresentaram significância em relação à adesão à triagem da TB. Melhores informações sobre a doença precisam ser fornecidas aos contatos de pacientes com TB para melhorar sua saúde, comportamento e reconhecimento de possíveis sintomas da tuberculose ${ }^{32}$.

Nas ações e práticas referentes ao controle da TB, a sociedade civil necessita ser esclarecida sobre a doença, bem como, deve ser explicitada sua corresponsabilidade dentro de todo o contexto da tuberculose. Ressalta-se que educação em saúde e, orientações sobre prevenção da tuberculose são essenciais ${ }^{33}$.

Neste estudo, verificou-se que os contatos de TB do DS II desconhecem a forma de transmissão da tuberculose e, a necessidade de serem avaliados e da realização dos exames solicitados. Dos entrevistados, $62,1 \%$ afirmaram que os profissionais de saúde não explicaram sobre tuberculose e apenas $55 \%$ relatam terem sido convidados para ser avaliados. Por estarem inseridos nas iniquidades sociais, muitas vezes, o empoderar possivelmente trará consigo indivíduos contatos mais conscientes quanto à TB, contribuindo para a detecção precoce dos casos, bem como para responsabilização, participação no processo e redução da incidência da tuberculose por interrupção na cadeia de transmissão da doença. A comunidade ter conhecimento sobre a TB pode influenciar nos resultados e corroborar para a eliminação da doença.

Durante as visitas domiciliares, para escuta dos indivíduos contatos e coleta das informações, identificou-se que, no que concerne aos exames preconizados pelo PNCT, os profissionais não seguem o padrão estabelecido. É sabido que cada pessoa necessita ser vista em sua individualidade e de forma distinta, nas suas diferenças. Mas as condutas diversas, quando se referem aos exames ou avaliação dos contatos de tuberculose, podem acarretar em falhas nesta avaliação ou possível irregularidade na condução dos casos de tuberculose pelo profissional da ESF, mesmo havendo as diretrizes já preconizadas. Vale destacar o baixo percentual encontrado de indivíduos contatos examinados que tiveram a Isoniazida prescrita.

As metas do Plano Nacional, que preconiza o Brasil livre da tuberculose como Problema de Saúde Pública, possuem três pilares essenciais. Os dois primeiros visam à atenção ao paciente 
e ao contexto social. O fluxograma estabelecido categorizado entre menores e maiores de dez anos, propicia uma excelente condução dos $\operatorname{cas}^{34}{ }^{34}$. Contudo, a partir dos achados apresentados neste estudo, reforça-se a necessidade de análise criteriosa sobre as condições sociais a serem consideradas tanto nas orientações gerais de promoção e prevenção, como na orientação dos exames a serem realizados e dos possíveis tratamentos.

Há possibilidade de rastreio adequado da ILTB, troca de informações a respeito da doença e estreitamento dos vínculos entre profissional e paciente durante as visitas e consultas. Realizar os exames preconizados é o momento inicial para avaliar a necessidade do tratamento da ILTB. Para tal, ressalta-se a necessidade de examinar o contato, compreender sua realidade e orientá-lo sobre a tuberculose. Dessa forma, haverá informação circulante, mais chances de adesão aos exames solicitados e, aos possíveis tratamentos profiláticos, e agentes multiplicadores sobre tuberculose dentro das comunidades.

Este estudo apresenta algumas limitações relacionadas ao registro das informações contidas nos dados secundários. No Sinan, não constam as idades e nomes dos contatos domiciliares, apenas o quantitativo. Dessa forma, alguns casos índices sorteados, que possuíam mais de dois contatos, muitas vezes eram inelegíveis devido à idade menor que 10 anos ou porque havia a incerteza de quais pessoas foram registradas ou investigadas.

Ressalta-se que todos os domicílios visitados receberam orientações quanto à tuberculose. Os indivíduos contatos que ainda não haviam sido examinados ou não tinham realizado os exames foram encaminhados para as suas Unidades de Saúde com conhecimento prévio dos profissionais das ESF. As informações e os dados dos domicílios nos quais foram encontradas inconsistências entre número de casos registrados e os que realmente residem foram repassadas para atualização no Sinan junto à Vigilância Epidemiológica do Distrito Il.

Este estudo tem potencial de contribuição para subsidiar gestores e profissionais acerca da importância da formação da consciência coletiva sobre tuberculose e da necessidade da avaliação e solicitação dos exames profiláticos dos contatos. Para que essas ações aconteçam de forma efetiva, a captação e o acompanhamento dos contatos são processos fundamentais a serem desenvolvidos. Recomenda-se também as atualizações em ILTB junto aos profissionais de saúde, fortalecendo os vínculos e a percepção sobre a importância do acompanhamento dos contatos de TB.

\section{REFERÊNCIAS}

1. Forrellad MA, Klepp LI, Gioffré A, Sabio y García J, Morbidoni HR, de la Paz Santangelo M, et al. Virulence factors of the Mycobacterium tuberculosis complex. Virulence, Buenos Aires. 2013;4(1):3-66. http://dx.doi. org/10.4161/viru.22329. PMid:23076359.

2. World Health Organization. Global tuberculosis report 2018. Geneva: WHO; 2018.

3. Brasil. Ministério da Saúde. Secretaria de Vigilância em Saúde. Departamento de Vigilância das Doenças Transmissíveis. Brasil Livre da Tuberculose: Plano nacional pelo fim da tuberculose como problema de saúde pública. Brasília: Ministério da Saúde, 2017.

4. Ramos J, Wakoff-Pereira MF, Cordeiro-Santos M, Albuquerque MFM, Hill PC, Menzies D, et al. Knowledge and perceptions of tuberculosis transmission and prevention among physicians and nurses in three Brazilian capitals with high incidence of tuberculosis. J Bras Pneumol. 2018;44(2):168-170.

5. Organização Pan-Americana da Saúde. Progresso para acabar com a tuberculose nas Américas deve ser acelerado, afirma novo relatório da OPAS [Internet]. 2018 [citado em 2019 jun 1]. Disponível em: https:// www.paho.org/bra/index.php?option=com_content\&view=article\&id=5768:progresso-para-acabarcom-a-tuberculose-nas-americas-deve-ser-acelerado-afirma-novo-relatorio-da-opas\&ltemid=812

6. Brasil. Secretaria de Vigilância em Saúde. Ministério da Saúde. Situação epidemiológica e estratégias de prevenção, controle e eliminação das doenças tropicais negligenciadas no Brasil, 1995 a 2016 [Internet]. Brasília: Ministério da Saúde; 2018 [citado em 2019 jun 1]. (Boletim Epidemiologico, 49). Disponível em: portalms.saude.gov.br/images/pdf/2018/setembro/05/2018-041.pdf

7. Castillo RG, Jimenez GT. Epidemiologia de la tuberculosis. Rev Med Hosp Gen (Mex). 1982;45(5-6):164-7.

8. Rieder HL. Bases epidemiológicas do controle da tuberculose. Trad. José Miguel Carvalho. Lisboa: DireçãoGeral da Saúde; 2001. p. 168. 
9. Gazetta CE, Ruffino-Netto A, Pinto Neto JM, Santos ML, Cury MR, Vendramini SH, et al. Investigation of tuberculosis contacts in the tuberculosis control program of a medium-sized municipality in the southeast of Brazil in 2002. J Bras Pneumol. 2006;32(6):559-65. http://dx.doi.org/10.1590/S1806-37132006000600014. PMid:17435907.

10. Freire DN, Bonametti AM, Matsuo T. Diagnóstico precoce e progressão da tuberculose em contatos. Epidemiol Serv Saude. 2007;16(3):155-63.

11. Brasil. Ministério da Saúde. Secretaria de Vigilância em Saúde. Departamento de Vigilância das Doenças Transmissíveis. Protocolo de vigilância da infecção latente pelo Mycobacterium tuberculosis no Brasil. Brasília: Ministério da Saúde; 2018. p. 32.

12. Shivaramakrishna HR, Frederick A, Shazia A, Murali L, Satyanarayana S, Nair SA, et al. Isoniazid preventive treatment in children in two districts of South India: does practice follow policy? Int J Tuberc Lung Dis. 2014;18(8):919-24. http://dx.doi.org/10.5588/ijtld.14.0072. PMid:25199005.

13. González-Ochoa E, Brooks JL, Matthys F, Calisté P, Armas L, Van der Stuyft P. Pulmonary tuberculosis case detection trough fortuitous cough screening during home visits. Trop Med Int Health. 2009;14(2):131-5. http://dx.doi.org/10.1111/j.1365-3156.2008.02201.x. PMid:19236664.

14. Lemos AC, Matos ED, Pedral-Sampaio DB, Netto EM. Risk of tuberculosis among household contacts in Salvador, Bahia. Braz J Infect Dis. 2004;8(6):424-30. http://dx.doi.org/10.1590/S1413-86702004000600006. PMid:15880233.

15. Akolo C, Adetifa I, Shepperd S, Volmink J. Treatment of latent tuberculosis infection in HIV infected persons. Cochrane Database Syst Rev. 2010;1(1):1-37. http://dx.doi.org/10.1002/14651858.CD000171. pub3. PMid:20091503.

16. Dowell J, Hudson H. A qualitative study of medication-taking behaviour in primary care. Fam Pract. 1997;14(5):369-75. http://dx.doi.org/10.1093/fampra/14.5.369. PMid:9472370.

17. Teixeira PR, Paiva V, Shimma E. Tá difícil de engolir? Experiências de adesão ao tratamento anti-retroviral em São Paulo. São Paulo: Copidart; 2000.

18. Bertolozzi MR, Nichiata LYI, Takahashi RF, Ciosak SI, Hino P, Val LF, et al. Os conceitos de vulnerabilidade e adesão na Saúde Coletiva. Rev Esc Enferm. 2009;43(2):1326-30. http://dx.doi.org/10.1590/S008062342009000600031.

19. Queiroz AAR, Dantas DNA, Lopes RH, Menezes RMP, Enders BC, Arcêncio RA. Conhecimento das famílias de pacientes com tuberculose sobre a doença: estudo transversal. Cogitare Enferm. 2016;21(1):1-9. http:// dx.doi.org/10.5380/ce.v21i1.42136.

20. Ayres JRCM, França IJ, Calazans, GJ, Saletti Filho, HC. O conceito de vulnerabilidade e as práticas de saúde: novas perspectivas e desafios. In: Czeresnia D, Freitas CM, organization. Promoção da saúde: conceitos, reflexões, tendências. Rio de Janeiro: Fiocruz; 2003. p.117-140.

21. Procópio MJ, Hijjar MA, Porto OM. Tuberculose. In: Procópio MJ. Prevenção \& Controle. Rio de Janeiro: Quattri Desenhos; 2008. p. 40-55.

22. Instituto Brasileiro de Geografia e Estatística [Internet]. Pernambuco: IBGE; 2015 [citado em 2015 jan 20]. Disponível em: http://www.ibge.gov.br/home/

23. Recife. Secretaria de Saúde. Distritos Sanitários em Recife. Oito Distritos [Internet]. 2014 [citado em 2015 mar 20]. Disponível em: http://www2.recife.pe.gov.br/servico/estrutura-da-vigilancia-sanitaria

24. San Pedro A, Oliveira RM. Tuberculose e indicadores socioeconômicos: revisão sistemática da literatura. Rev Panam Salud Publica. 2013;33(4):294-301. http://dx.doi.org/10.1590/S1020-49892013000400009. PMid:23698179.

25. Fox GJ, Loan LP, Nhung NV, Loi NT, Sy DN, Britton WJ, et al. Barriers to adherence with tuberculosis contact investigation in six provinces of Vietnam: A nested case-control study. BMC Infect Dis. 2015;15(1):1-8. http://dx.doi.org/10.1186/s12879-015-0816-0. PMid:25886411.

26. Rodrigues ILA, Monteiro LL, Pacheco RHB, Silva SED. Abandono do tratamento de tuberculose em coinfectados TB/HIV. Rev Esc Enferm. 2010;44(2):383-7. http://dx.doi.org/10.1590/S008062342010000200020.

27. Trajman A, Long R, Zylberberg D, Dion MJ, Al-Otaibi B, Menzies D. Factors associated with treatment adherence in a randomised trial of latent tuberculosis infection treatment. Int J Tuberc Lung Dis. 2010;14(5):551-9. PMid:20392347. 
28. Brasil. Ministério da Saúde. Secretaria de Vigilância em Saúde. Nota Informativa nº 08, de 2014 CGPNCT/ DEVEP/SVS/MS. Recomendações para controle de contatos e tratamento da infecção latente da tuberculose na indisponibilidade transitória do Derivado Proteico Purificado. Diário Oficial da União [Internet], Brasília, Jul. 2015 Disponível em: http://portal.saude.pe.gov.br/sites/portal.saude.pe.gov.br/ files/nota_informativa_ppd.pdf.

29. Young KH, Ehman M, Reves R, Maddox BLP, Khan A, Chorba TL, et al. Tuberculosis contact investigations: United States of America, 2003-2012. MMWR Surveill Summ. 2016;64(50):369-74.

30. Okuonghae D, Omosigho S. Determinants of TB Case Detection in Nigeria: A survey department of mathematics. Glob J Health Sci. 2010;2(2):1-6. http://dx.doi.org/10.5539/gjhs.v2n2p123.

31. Thanh TH, Ngoc SD, Viet NN, Van HN, Horby P, Cobelens FG, et al. A household survey on screening practices of household contacts of smear positive tuberculosis patients in Vietnam. BMC Public Health. 2014;14(1):1-13. http://dx.doi.org/10.1186/1471-2458-14-713. PMid:25015682.

32. Gebremedhin BG, Wondmu GA. Household Contact Screening adherence among Tuberculosis Patients in Northern Ethiopia. PLoS One. 2015;10(5):1-8.

33. Curto M, Scatena LM, Andrade RLP, Palha PF, Assis EG, Scatolín BE, et al. Tuberculosis control: patient perception regarding orientatíon for the cornmunity and community partícípation. Rev Lat Am Enfermagem. 2010;18(5):983-9. http://dx.doi.org/10.1590/S0104-11692010000500020. PMid:21120419.

34. Brasil. Ministério da Saúde. Secretaria de Vigilância em Saúde. Coordenação Geral de Desenvolvimento da Epidemiologia em Serviços. Guia de Vigilância em Saúde: volume único. 2. ed. Brasília: Ministério da Saúde; 2017. p. 705. 